\title{
SHADOW HAND
}

\author{
Dhruv Sharma ${ }^{1 *}$, Kirti Tokas ${ }^{2}$, Aviral Puri ${ }^{3}$, Krishna Sharda ${ }^{4}$ \\ *1,2,3,4 Dronacharya College of Engineering
}

*Corresponding Author: -

\begin{abstract}
: -
The shadow hand is an advanced humanoid hand that uses hand movements and trajectory tracking to control the motion of shadow hand. The shadow hand will be integrated with 40 Air Muscles or 20 DC motors with the help of which it will move. The muscles will be in accordance allowing the hand to be used around soft or fragile objects. The hand will be shaped with touch sensors on finger tips to offer high sensitivity. This shadow hand robot has its various applications in household work, telepresence operations, bomb disarmament, military and medical purposes. Through this shadow hand bot, we would also be able the problems faced by handicapped people.
\end{abstract}

Keywords: Actuation, Degree of Freedom, Embedded, EtherCat, Pneumatics, Tactile Sensors, Trajectory,

\section{(c) $(\$)$}




\section{INTRODUCTION}

The shadow hand is a human-like robot hand designed to do works that are either dangerous for any human to do or to save their time from any kind of extra work. Whether you are at house, doing surgical operations, at field disarming a bomb or at a radiation full environment to collect specimens, this robot hand will come to your rescue and save you from the threat of losing your life.

The term robot has come from the word "robota", which is a Czech word. It is widely interpreted as forced labor. Robots are sketched for repetitive work. They are widely used to do tasks which are burdensome, dangerous or boring to human beings. The shadow hand is advancement in the robotics field. The hand provides movements that are similar to the kinematics and proficiency of the human hand. The shadow hand is an independent system.-all sensing and actuation is fixed in the hand itself.

Now in this section we will deal with all of the below:

- Design

- Control system

- Software

- Mechanical profile

\subsection{DESIGN}

The shadow hand will be sketched to be similar to the average human hand as much as possible. The shadow hand will have 24 joints and its degree of freedom is 20 , which is much more than the human hand. The four fingers of the hand will contain two one-axis joints which will join the distal phalanges, intermediate phalanges and proximal phalanges and also one universal joint which will join the phalanges to the metacarpal similar to the structure of a human hand. For the curling movement of palm, the little finger is provided with an extra one-axis joint on the metacarpal. A one-axis joint is provided to the thumb to connect the distal phalanges to the proximal phalanges, and similar to the four fingers, it also has one universal joint connecting the thumb to the metacarpal. One one-axis joint is also provided at the bottom of metacarpal to connect the metacarpal and carpal together to give a better palm curling movement. The wrist contains two joints which helps in the flex and adduct movement of the hand similar to the function of pectorals in human. The hand can be made in both electric motor driven and pneumatic muscle driven form. The motor driven hand can be controlled by 20 DC motors attached to the forearm in contrast to which the muscle driven arm can be operated by 20 agonist pairs of air muscles attached to the forearm. The motor hand will have force sensors for each degree of freedom whereas the muscle hand will involve pressure sensors for each muscle.

Below is the table with the detail sketch of the shadow hand.

Table:

\begin{tabular}{|c|c|c|c|}
\hline Joint & Connects & Range & Muscle Type \\
\hline \multicolumn{4}{|c|}{ First, Middle, Ring finger } \\
\hline 1 & Distal - Middle & $-20-+90$ & \multirow{2}{*}{ Coupled pair } \\
\hline 2 & Middle - Proximal & $0-+90$ & \\
\hline 3 & $\begin{array}{l}\text { Proximal } \\
\text { Knuckle }\end{array}$ & $-20-+90$ & Pair \\
\hline 4 & Knuckle - Palm & $-25-+25$ & Single with Spring \\
\hline \multicolumn{4}{|c|}{ Little Finger } \\
\hline 1 & Distal - Middle & $-20-+90$ & \multirow{2}{*}{ Coupled pair } \\
\hline 2 & Middle - Proximal & $0-+90$ & \\
\hline 3 & $\begin{array}{l}\text { Proximal } \\
\text { Knuckle }\end{array}$ & $-20-+90$ & Pair \\
\hline 4 & $\begin{array}{l}\text { Knuckle } \\
\text { Metacarpal }\end{array}$ & $-25-+25$ & Single Spring with \\
\hline 5 & Metacarpal - Palm & $0-+40$ & Pair \\
\hline \multicolumn{4}{|c|}{ Thumb } \\
\hline 1 & Distal - Middle & $-20-+90$ & Pair \\
\hline 2 & Middle - Proximal 1 & $-40-+40$ & Pair \\
\hline 3 & Middle - Proximal 2 & $-15-+15$ & Pair \\
\hline 4 & Proximal - Palm 1 & $0-+80$ & Pair \\
\hline 50 & Proximal - Palm 2 & $-60-+60$ & Pair \\
\hline \multicolumn{4}{|c|}{ Wrist92 } \\
\hline 1 & Palm - Wrist & $-55-+45$ & Pair \\
\hline 2 & Wrist - Forearm & $-30-+10$ & Pair \\
\hline
\end{tabular}

The thumb has 5 degrees of freedom and 5 joints.

Each finger has 3 degrees of freedom and 4 joints. 


\subsection{CONTROL SYSTEM}

The controlling system is different in both motor driven and muscle driven hand. The motor driven hand will use the smart motor actuation system whereas the muscle driven will use the pneumatic air muscle actuation system. The smart motor will amalgamate motor force and position control electronics, gear box, motor drive electronics, force sensing and communications into a dense module, 20 of which will be loaded at the hand base. The pneumatic air muscle system will combine pressure and position sensing electronics, valve driving electronics, manifold and communication for 80 valves in its own dense base.

\subsection{SOFTWARE}

The software system of the shadow hand robot is rooted to ROBOT OPERATING SYSTEM (ROS), because of which simulation, configuration and control of the hand is executed.

The hand uses an EtherCAT bus. EtherCAT is the abbreviation used for Ethernet for Control Automation Technology. It is a Ethernet built field bus with a $100 \mathrm{mbps}$ speed. It is widely used in many systems as it makes the hand compatible with systems that are EtherCAT/ROS compatible. A powerful multi-core PC with a standard Ethernet port is needed for the successful working of EtherCAT bus plus ROS. An embedded Ethernet interface option permits direct access to robot data and configuration by TCP/IP communication.

\subsection{MECHANICAL PROFILE}

The structure of the shadow hand is similar to that of the human forearm in length. The table given below describes the basic structure of the shadow hand.

Table:

\begin{tabular}{|l|l|}
\hline Finger length from tip of finger to middle of knuckle & $100 \mathrm{~mm}$ \\
\hline Thumb length & $102 \mathrm{~mm}$ \\
\hline Palm length from middle knuckle to wrist axis & $99 \mathrm{~mm}$ \\
\hline Palm thickness & $22 \mathrm{~mm}$ \\
\hline Palm width & $84 \mathrm{~mm}$ \\
\hline Thumb base thickness & $34 \mathrm{~mm}$ \\
\hline Forearm base to wrist axis & $434 \mathrm{~mm}$ \\
\hline
\end{tabular}

Weight: The weight of the shadow hand is different for both motor driven hand and muscle driven hand. The weight of motor driven hand is greater than that of muscle driven hand. The weight in case of motor driven hand is $4.2 \mathrm{~kg}$ which constitutes of hand, forearm and motors. And in case of muscle driven hand is $3.9 \mathrm{~kg}$ which involves hand, sensors, valve manifold and muscles.

Speed: Some variations in the movement speeds between the parts of the hand are noticed. Moreover, the speed of the hand varies with the movements performed. Nevertheless, the general movement observed is about half the speed to that of a human hand. Although the time taken by the hands for performing movements is observed as: (approx.)

\begin{tabular}{|l|l|}
\hline SHADOW HAND & TIME (sec.) \\
\hline MOTOR & 1.0 \\
\hline MUSCLE & 0.2 \\
\hline
\end{tabular}

Material: The hand is a union of metals and plastics which involve the following:

\begin{tabular}{|l|l|}
\hline Forearm bone & Steel \\
\hline Palm & Acetyl, aluminum, polycarbonate \\
\hline Fingers & Acetyl, aluminum, polycarbonate fingernails and polyurethane flesh \\
\hline Base & Acetyl, rubber, brass, cork \\
\hline
\end{tabular}

\section{COMMUNICATIONS}

The version of the Hand uses an EtherCAT bus. EtherCAT (Ethernet for Control Automation Technology) is a 100Mbps Ethernet-based fieldbus. It is currently used in a number of systems, such as Willow Garage's PR2 robot, making these versions of the Hand compatible with the PR2, and any other research or industrial control systems that are EtherCAT/ROS compatible.

The EtherCAT bus plus ROS requires a powerful multi-core PC (supplied) with a standard Ethernet port. The EtherCAT protocol used by the hand is simple since the position control loop happens in the host (documentation supplied)

\subsection{Motor Hand Features}

- Change torque control PID values

- Change operational limits such as force and temperature cut-outs 
- Reset motors

- Adjust data transmission rates for motors and tactile sensors

- Track error and status indicators from the components

- Download new firmware into the Smart Motor modules

\subsection{Control}

As standard, the EtherCAT Hand implements a position control strategy in the host PC. Other Control algorithms can be used as much more complex control strategies can be implemented, fusing information from joint and tactile sensors and even visual signals via ROS. In the Motor Hand, the torque loop is closed inside the motor unit at $5 \mathrm{kHz}$. The PID settings for this loop can be changed in real time. Alternatively, new firmware can be downloaded into the motor units if you require a different control strategy, or a new version is available from Shadow. All other control loops run at $1 \mathrm{kHz}$ through the host.

In the Air Muscle Hand there are no internal control loops and all loops run at $1 \mathrm{kHz}$ from the host. New firmware can be downloaded into the valve drivers if you require a different control strategy, or a new version is available from Shadow. The PID controllers are set up in the configuration or boot phase of the system, can be changed on the fly, and can be configured to operate from sensor data and from user-supplied values, permitting control of joint position, force, Air Muscle pressure, or user-supplied parameters.

\subsection{Micro-controllers}

Microchip PIC18Fxx80 micro-controllers are used for embedded control throughout the robot system, except on the palm, where a PIC32 is used; and on the Pressure Sensor Tactile (PSTs), where PSoCs are used. The firmware is available upon request by customer under Non-Disclosure Agreement (NDA). All micro-controllers are connected to the internal bus and can be accessed via the EtherCAT interface.

\section{Applications}

-Highly Sensitive medical surgeries.

-Bomb disposal vehicle.

-Household works.

-High tension broken wires solver.

-Serious chemical reaction like Fission and Fusion.

\section{References}

[1].DB,http://www.erato.atr.co.jp/db/home.html,

[2].Michael Gleicher, "Motion editing with spacetime constraints,"1997 Symposium on Interactive 3D Graphics, pp. 139-148, April 1997.

[3].Michael Gleicher, "Retargeting motion to new characters," Proceedings of SIGGRAPH 98), pp. 33-42, July 1998.

[4].Vicon Motion Systems, "http://www.vicon.com/,"

[5].Shadow Dexterous Hand Technical Specification, "Shadow robot company"

[6].Adapting Human Motion for the Control of a Humanoid Robot, "Nancy S. Pollard, Jessica K. Hodgins, Marcia J. Riley, Christopher G. Atkeson" 